\title{
Hand-assisted sputum excretion can effectively reduce postoperative pulmonary complications of esophageal cancer
}

\author{
Wei Wang ${ }^{1 \#}$, Qi Liu ${ }^{1 \#}$, Yongkui Yu ${ }^{1}$, Haibo Ma ${ }^{1}$, Lei Xu ${ }^{2}$, Ruixiang Zhang ${ }^{1}$, Haibo Sun ${ }^{1}$, Zongfei Wang ${ }^{1}$, \\ Yan Zheng ${ }^{1}$, Peinan Chen ${ }^{1}$, Shilei Liu ${ }^{1}$, Funa Yang ${ }^{1}$, Qiyun Zou ${ }^{1}$, Aiying Sun ${ }^{1}$, Xiaofei Chu ${ }^{1}$, \\ Chenfang Gong ${ }^{3}$, Wenqun Xing ${ }^{1}$
}

${ }^{1}$ Department of Thoracic Surgery, The Affiliated Cancer Hospital of Zhengzhou University, Henan Cancer Hospital, Zhengzhou, China; ${ }^{2}$ Department of Thoracic Surgery, Cancer Hospital Chinese Academy of Medical Sciences, Beijing, China; ${ }^{3}$ Respiratory Medicine, Children's Hospital of Zhengzhou University, Zhengzhou, China

Contributions: (I) Conception and design: W Xing; (II) Administrative support: W Xing, F Yang; (III) Provision of study materials or patients: Q Liu, H Ma, R Zhang, H Sun, Z Wang, Y Zheng, P Chen, S Liu, Q Zou, A Sun, X Chu; (IV) Collection and assembly of data: W Wang, C Gong; (V) Data analysis and interpretation: Y Yu, L Xu; (VI) Manuscript writing: All authors; (VII) Final approval of manuscript: All authors.

\#These authors contributed equally to this work.

Correspondence to: Wenqun Xing. Department of Thoracic Surgery, The Affiliated Cancer Hospital of Zhengzhou University, Henan Cancer Hospital, Zhengzhou 450008, China. Email: zlyyxwk2012@163.com.

Background: This study explores whether postoperative hand-assisted expectoration can reduce postoperative pulmonary complications (PPCs) in patients with esophageal cancer.

Methods: A retrospective analysis was performed on 543 patients undergoing radical esophageal cancer (EC) surgery in our hospital from October 2018 to August 2019, 156 of whom received postoperative handassisted sputum excretion (pulmonary rehabilitation, PR) and 387 of whom who did not receive postoperative hand-assisted sputum excretion (no pulmonary rehabilitation, NPR). Because the clinical characteristics of the two groups were not balanced, we used propensity score matching (PSM) to account for the variable factors of age, gender, body mass index (BMI), chronic respiratory comorbidity, smoking index, operation time, operation method, pathological stage. The main observation index used was PPCs.

Results: Among these 543 patients, 365 were male (67.2\%), while 178 were female (32.8\%). The age ranged from 30 to 82 years, with an average of $63.6 \pm 7.5$ years old. In all, 342 patients (63\%) underwent video-assisted thoracic surgery (VATS) surgery, while 201 patients (37\%) underwent thoracotomy. Furthermore, 72 patients in the $\mathrm{PR}$ group received preoperative rehabilitation training and postoperative hand-assisted sputum excretion (combination pulmonary rehabilitation, CPR), while 87 patients only received postoperative hand-assisted sputum excretion (postoperative pulmonary rehabilitation, PPR). The patients in the PR group and the NPR group were uneven in terms of clinical characteristics, and we performed PSM as a result. After matching, PPC incidence in patients in the PR group was lower than that in the NPR group $(\mathrm{P}<0.05)$.

Conclusions: Our results show that hand-assisted sputum excretion after EC surgery can reduce PPCs.

Keywords: Hand-assisted sputum excretion; esophagectomy; pulmonary rehabilitation; postoperative pulmonary complications (PPCs); chest physical therapy

Submitted May 15, 2020. Accepted for publication Aug 18, 2020.

doi: 10.21037/apm-20-1267

View this article at: http://dx.doi.org/10.21037/apm-20-1267 


\section{Introduction}

In China, esophageal cancer (EC) is a common malignant tumor of the digestive system, and surgery is still the main treatment $(1,2)$. Postoperative pulmonary complications (PPCs) are the most common type to occur after esophagectomy, accounting for $28.9-50 \%$ of all complications (3-5) In thoraco-abdominal surgery, PPCs increase morbidity and mortality, extend hospital stay, and raise medical expenses $(6,7)$.

With the development of minimally invasive technology and the application of the rapid rehabilitation concept, perioperative physiotherapy of $\mathrm{EC}$ has also become increasingly important (8-10), with postoperative chest physical therapy being an indispensable part of the postoperative recovery of esophageal cancer (10).

Most of the reports in the literature discuss preoperative inspiratory muscle training $(11,12)$, aerobic exercise (13), and early postoperative activities (14), but few studies have examined if chest physical therapy can effectively prevent or treat PPCs of EC (15).

A meta-analysis showed that perioperative interventions to reduce PPCs in adult with non-cardiac surgery: use of enhanced recovery after surgery pathways $(16,17)$, postoperative continuous positive airway pressure noninvasive ventilation $(18,19)$, lung protective intraoperative ventilation $(20,21)$, epidural analgesia $(22,23)$, and goal directed haemodynamic therapy (24-26). Nakamura et al. (27) found through the study of 184 patients with EC that the incidence of PPCs in patients who did not receive chest physical therapy was four times that of patients who received chest physical therapy. If the patient cannot effectively remove secretions or has a weak cough, recovery is exceedingly difficult. The purpose of this study then was to determine whether postoperative hand-assisted sputum reduction could reduce PPCs after EC surgery and thus promote patient recovery.

We present the following article in accordance with the STROBE reporting checklist (available at http://dx.doi. org/10.21037/apm-20-1267).

\section{Methods}

\section{Participant information}

A retrospective analysis was performed on 543 patients who underwent radical esophagectomy in a tertiary oncology hospital from October 2018 to August 2019. The study was conducted in accordance with the Declaration of
Helsinki (as revised in 2013). The study protocol was approved by the Ethics Committee of the Affiliated Cancer Hospital of Zhengzhou University (2014ys38), and the requirement for informed consent was waived because of the retrospective nature of the research. We collected all clinical, postoperative complication, and pathological data from the LinkDoc database. Patients only with pulmonary complication, such as, pleural effusion, atelectasis, pneumonia, respiratory failure, respiratory distress, enrroled in our study, patients with non-pulmonary complications, such as anastomosis leak, myocardial infarction, incision infection, etc., were excluded. Patients who underwent EC resection during the past 10 months were then divided into the two following groups: 156 patients who received postoperative hand-assisted sputum excretion (PR), and 387 patients who did not receive postoperative hand-assisted sputum excretion [no pulmonary rehabilitation (NPR)].

\section{Preoperative preparation and treatment}

All patients underwent blood routine, liver and kidney function, chest and abdomen computed tomography (CT), electrocardiogram (ECG), pulmonary function, esophagography, gastroscopy, and other related examinations before operation. All the patients were confirmed by pathology. All patients received respiratory tract treatment with ambroxol and doxofylline.

\section{Surgical approach}

Conventional anesthesia with double-lumen endotracheal intubation was completed, and the surgical approach included video-assisted thoracic surgery (VATS) and thoracotomy. There were 201 cases of thoracotomy, including 16 cases of Sweet esophagectomy, 101 cases of McKeown esophagectomy, and 84 cases of left thoracotomy with cervical anastomosis; meanwhile, there were 342 cases of VATS surgery.

\section{Rehabilitation plan}

After admission, patients in the PR and NPR groups routinely received pulmonary rehabilitation education. Patients in the PR group underwent preoperative rehabilitation and performed postoperative hand-assisted sputum excretion aided by a physical therapist in the hospital. The NPR group did not receive any preoperative rehabilitation or postoperative chest physical therapy. The 
specifics of the preoperative rehabilitation program were as follows:

(I) Respiratory muscle training: this included abdominal breathing and lip constriction breathing with the help of an inspiratory muscle trainer.

(II) Expiratory flow rate training: patients clenched their fists and lifted their arms forward. Next, they drew their fists to their chests while inhaling and then vigorously extended their fists while exhaling. This was repeated 5-8 times per group, and with 2-3 groups a day.

(III) Cough method training: this consisted of taking a deep breath, holding the breath, and coughing hard.

(IV) Boarding training: this was accompanied by a physical therapist and involved adjusting breathing rhythm during exercise.

(V) Practice: after the patients had learned the breathing exercises from our department, they practiced them independently.

The above preoperative rehabilitation training program was carried out 3-7 days before the operation.

The postoperative rehabilitation program proceeded as follows:

(I) Early activity: on the first day after surgery, the physiotherapist clinically assessed the patients' stable vital signs and encouraged patients to start sitting up next to bed as soon as possible, to stand up, and to walk in place.

(II) Respiratory muscle training: postoperative patients started deep breathing training based on abdominal breathing and lip contraction breathing as soon as possible after waking up.

(III) Effective pain relief: this was implemented according to the visual analog score (VAS) scale for pain relief.

(IV) Physiotherapy: according to the patient's condition, physiotherapists applied airway clearance techniques (ACT) including active cycle of breathing techniques (ACBT), mechanically assisted sputum discharge, and buckle vibrations.

(V) Hand-assisted sputum excretion: if the patient could not effectively remove respiratory secretions after surgery, the physical therapist enabled coughing and expectorating with hand assistance. The specific operation proceeds as follows. With the patient sitting, the physiotherapist uses his or her right hand at the level of the patient's navel; the patient is told to commence abdominal breathing, holding breath for 1-3 s after deep inhalation. While the patient attempts coughing, the physiotherapist pushes his or her right hand on the side of and above the abdomen.

(VI) After surgery, all patients also received respiratory tract treatment with ambroxol and doxofylline.

This technique can be further divided into two types.

Hand-assisted sputum excretion method by controlling cough: the patients takes three deep breaths. After the first two deep breaths, they hold their breath for three seconds and then deeply exhale. At the same time, the therapists pushes and presses the upper part of the abdomen with their hands. Hand-assisted sputum excretion should be used during the third deep breath. The first two deep breaths can increase the vital capacity of the final cough, and the action of pushing and pressing the upper part of the abdomen during exhalation and cough can increase the intrapleural pressure and intra-abdominal pressure, speed up air flow, and promote the movement of sputum.

Hand-assisted sputum excretion method by continuous cough: first, patients inhale and cough normally. Second., patients inhale and cough at moderate intensity. Finally, patients inhale deeply and cough intensely. Hand-assisted sputum excretion should be used for each cough; it used for coughing with poor inhalation and helpful for further improving the intensity of the cough.

When using the techniques above, patients should wear a chest and abdomen belt, grasp the respiratory rate and cough rhythm of the patients or follow the slogan of the physical therapists when using the hand-assisted method, and control the intensity of pushing and pressing, from gentle to hard.

\section{Observation index}

The main observation index was PPCs. Evaluation of PPCs was done using the Clavien-Dindo classification (CDC) scoring system which includes pneumonia, atelectasis, pleural effusion, and respiratory failure. A description of the assessment for each of these aspects follows below.

Pneumonia: (I) X-ray- or CT-confirmed and requiring antibiotic treatment; (II) clinical symptoms including body temperature $>38$ or $<35.5^{\circ} \mathrm{C}$; (III) emergence or increase of sputum production; (IV) white blood cell count (WBC) $\leq 4 \times 10^{9} \mathrm{~L}$ or $\mathrm{WBC} \geq 12 \times 10^{9}$. Pneumonia was confirmed if found positive on imaging findings and any one of the two clinical symptoms was present in the patient. 
Atelectasis: X-ray or CT-confirmed and requiring treatment with bronchoscopy.

Pleural effusions: X-ray or CT-confirmed and requiring treatment.

Respiratory failure: requiring reintubation $24 \mathrm{~h}$ after surgery, or more than 72 without extubation on the day of surgery.

\section{Statistical methods}

This study analyzed the data of two groups of patients. Categorical variable data are described numerically and as a percentage (\%). Chi-square test was used for statistical comparison. To reduce the impact of potential confounding factors, we performed propensity score matching (PSM). The matching variables were age, sex, body mass index (BMI), smoking index, chronic respiratory comorbidities, pre $\mathrm{FEV} 1 \%$, surgical approach, duration of surgery, and TNM stage. The matching method adopted caliper value matching, which could be replaced repeatedly during the matching process. The caliper value was 0.01 , and the matching ratio was $1: 1$. After PSM, two new subgroups were generated, and then the Chi-square test was used to compare the baseline characteristics of the two groups of patients. A P value $>0.05$ indicated that the two groups of patients were balanced in all factors. PSM used R language for operation, while the rest of the data analysis used SPSS 25 statistical software. A bilateral $\mathrm{P}$ value $<0.05$ was considered to indicate statistical significance.

\section{Results}

We performed a retrospective analysis of 543 patients who underwent radical esophagectomy from October 2018 to August 2019. There were 387 cases in the NPR group and 156 cases in the PR group; 72 patients received preoperative rehabilitation training and postoperative hand-assisted sputum excretion [combination pulmonary rehabilitation $(\mathrm{CPR})]$, and 87 patients received only postoperative handassisted sputum excretion (PPR). There were 365 males $(67.2 \%)$ and 178 females (32.8\%), with ages ranging from 30 to 82 years and an average of $63.6 \pm 7.5$ years. A total of 342 patients (63\%) underwent VATS surgery, and 201 patients (37\%) underwent thoracotomy (Table 1).

The two groups of patients were unbalanced in terms of clinical characteristics, and thus PSM was performed. After PSM, results showed that patients in the PR group had fewer PPCs than those in the NPR group $(\mathrm{P}<0.05)$ (Table 2).
The PPCs of the two groups before and after matching are shown in Table 3. The mortality rates within 30 days before and after matching were $1.02 \%$ and $2.44 \%$, respectively.

\section{Discussion}

PSM analysis results of these 543 cases of EC after operation showed that the incidence of PPCs in the PR group was lower than that in the NPR group.

Reports in the literature confirm the benefits of rapid rehabilitation in patients undergoing upper abdominal surgery $(28,29)$. Most of these studies evaluate the preventive effect of chest physiotherapy for EC resection or compare the postoperative effects of different techniques, including preoperative suction muscle training, aerobic exercise, early postoperative activities, etc. (11,30-32). These may reduce the risk of PPCs of esophageal cancer.

Perhaps due to the complexity of radical surgery for esophageal cancer, few studies have reported on chest physical therapy after EC surgery. In Lunardi et al.'s (33) retrospective analysis, patients with chest physical therapy after esophagectomy were found to have a lower incidence of pulmonary complications. This study also applied the assisted sputum excision technique of critical rehabilitation to the postoperative rehabilitation of patients with esophageal cancer.

In our study, there were 72 patients in the CPR group whom surgeons subjectively assessed to be at high risk for PPCs. Physical therapists performed preoperative rehabilitation training and postoperative chest physical therapy on these patients. There were 84 patients in the PPR group whom surgeons subjectively assessed to be at low risk for PPCs. These patients were given no preoperative rehabilitation training. However, when the patient appeared to be unable to effectively remove respiratory secretions after surgery, a physiotherapist began to actively intervene. Operations on EC can damage the lung tissue of patients and destroy the integrity of the patient's chest, intercostal muscles, diaphragm, and other areas, which reduces the affected lung's ventilation capacity. In addition, operation causes postoperative pain which leads to decreased breathing movement, reduces inspiratory volume, and weakens coughing.

In our case, two patients suffered injury to the recurrent laryngeal nerve during operation, resulting in hoarseness, incomplete vocal cord closure, and the inability to form an effective cough. We used the Huff technique combined with 
Table 1 Baseline characteristics of patients before propensity score matching

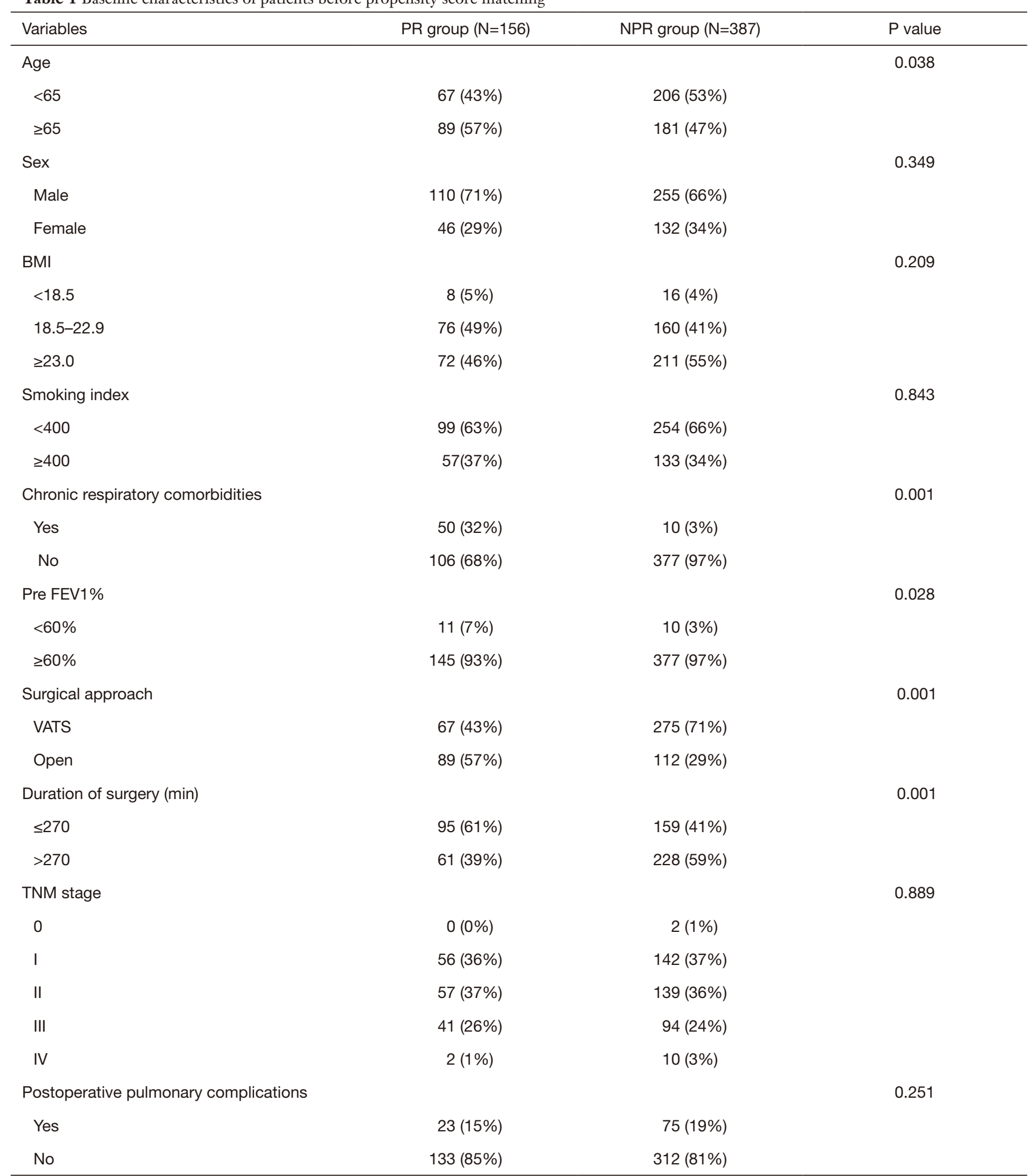

BMI, body mass index; VATS, video-assisted thoracic surgery; FEV1\%, forced vital capacity in the first second expressed as a percent of predicted. 
Table 2 Characteristics of patients after propensity score matching

\begin{tabular}{|c|c|c|c|}
\hline Variables & PR group ( $\mathrm{N}=101)$ & NPR group $(\mathrm{N}=101)$ & $P$ value \\
\hline$<65$ & $48(48 \%)$ & $48(48 \%)$ & \\
\hline$\geq 65$ & $53(52 \%)$ & $53(52 \%)$ & \\
\hline Sex & & & 1.000 \\
\hline Female & $32(32 \%)$ & $32(32 \%)$ & \\
\hline BMI & & & 0.126 \\
\hline$<18.5$ & $4(4 \%)$ & $0(0 \%)$ & \\
\hline $18.5-22.9$ & $45(45 \%)$ & $51(50 \%)$ & \\
\hline$<400$ & $73(72 \%)$ & $71(71 \%)$ & \\
\hline$\geq 400$ & $28(28 \%)$ & $30(30 \%)$ & \\
\hline Chronic respiratory comorbidities & & & 1.000 \\
\hline Yes & $6(6 \%)$ & $6(6 \%)$ & \\
\hline No & 95 (94\%) & 95 (94\%) & \\
\hline Pre FEV1\% & & & 0.621 \\
\hline$<60 \%$ & $1(1 \%)$ & $3(3 \%)$ & \\
\hline$\geq 60 \%$ & $100(99 \%)$ & $98(97 \%)$ & \\
\hline$>270$ & $48(48 \%)$ & $54(53 \%)$ & \\
\hline TNM stage & & & 0.657 \\
\hline I & $40(40 \%)$ & $40(40 \%)$ & \\
\hline II & $39(39 \%)$ & $32(32 \%)$ & \\
\hline III & $21(21 \%)$ & $28(28 \%)$ & \\
\hline Postoperative pulmonary complications & & & 0.036 \\
\hline Yes & $14(14 \%)$ & $27(27 \%)$ & \\
\hline No & $87(86 \%)$ & $74(73 \%)$ & \\
\hline
\end{tabular}

BMI, body mass index; VATS, video-assisted thoracic surgery; FEV1\%, forced vital capacity in the first second expressed as a percent of predicted. 
Table 3 Postoperative pulmonary complications before and after PSM

\begin{tabular}{lccccc}
\hline \multirow{2}{*}{ Outcomes } & \multicolumn{2}{c}{ Before PSM } & & After PSM \\
\cline { 2 - 5 } & PR $(n=23)$ & NPR $(n=75)$ & PR $(n=14)$ & 7 & NPR $(n=27)$ \\
\hline Pneumonia & 13 & 52 & 5 & 20 \\
Atelectasis & 7 & 15 & 1 & 4 \\
Pleural effusion & 2 & 3 & 1 & 1 \\
Respiratory failure & 1 & 5 & & 2 \\
\hline
\end{tabular}

PSM, propensity score matching; PR, pulmonary rehabilitation; NPR, no pulmonary rehabilitation; CPR, combination pulmonary rehabilitation; PPR, postoperative pulmonary rehabilitation.

wound support in these patients. The shear force generated during the exhalation of the Huff process can reduce the viscosity of the mucus $(34,35)$. In addition, the fast flow of air from strong exhalation causes the contraction of the chest wall and abdominal muscles to squeeze out the air, expelling the sputum in the distal airway. If the patient still had no energy to cough up the sputum, the Huff technique was combined with hand-assisted coughing.

An effective cough can help patients clear airway secretions in a timely fashion and reduce the incidence of pulmonary complications. When the patient is unable to effectively remove secretions after surgery, we often hear obvious wet rales, and the breathing rate will be faster, and severe cases may have dyspnea and irritability. Lunardi et al.'s (33) retrospective analysis found that postoperative respiratory training and $\mathrm{ACT}$ for EC could reduce the incidence of PPCs.

In our research, when the patient failed to remove respiratory secretions after surgery, PPCs usually occurred within 1-4 days after esophagectomy. When we performed hand-assisted coughing, generally combined with ACT (ACBT, deep breath training, oscillatory positive expiratory pressure, clapping and vibration), patients received treatment an average of at least once a day and about $20 \mathrm{~min}$ each time. One patient still experienced respiratory failure after hand-assisted coughing on the fourth day after surgery.

Some studies indicated that high-risk factors, including malnutrition, advanced age, FEV $1 \%<60 \%$, diffusing capacity for carbon monoxide (DLCO\%) $<60 \%$, smoking history, chronic respiratory comorbidities, thoracotomy, and long operation time can frequently lead to the occurrence of PPCs (36-38). Many studies recommend adequate preoperative cardiopulmonary function assessment, inspiratory muscle training, aerobic training, and resistance training, along with nutritional support and psychological intervention $(39,40)$. In our PR group, most patients were older than 65 , and had chronic respiratory comorbidities with thoracotomy, which is likely the reason why the incidence of PPCs was high. At present, most clinical pathways recommend that bedside sit-up on the first day after EC surgery should last longer than $2 \mathrm{~h}$, and that patients take a short walk (41). Hanada et al. (14) retrospectively analyzed 118 patients undergoing thoracoscopic esophagectomy; it was found that the incidence of postoperative atelectasis in the early active intervention group was significantly lower than that in the non-intervention group. Meanwhile, Bhatt et al. (42) found that bedside bicycle aerobic exercise for postoperative patients can reduce postoperative respiratory infections and postoperative hospital stays.

In our research, the two groups were combined for analysis because the sample size of the CPR and PPR groups was small. The results supported the effectiveness of hand-assisted sputum excretion. In one report on lung cancer, the combination of preoperative and postoperative pulmonary rehabilitation (PPR) was shown to significantly reduce the incidence of postoperative pneumonia. Fujimoto et al.'s (43) retrospective analysis of 15,146 patients after pneumonectomy found that the onset of pneumonia was less frequent in the combination group, as compared with the preoperative, postoperative, and no rehabilitation group; also, the postoperative group had a significantly lower incidence of pneumonia than the no rehabilitation group. Therefore, perioperative pulmonary rehabilitation is also feasible in esophagectomy $(40,44)$. Physiotherapists also play a key role in improving the cardiopulmonary function of surgery and controlling PPCs (45-47).

Some limitations to this study should also be addressed. This study is a retrospective study, and there were limited 
data for the evaluation scale. Our data could not be effectively compared using evaluations such as the sixminute walk test (6MWT), maximal inspiratory pressure (MIP), pulmonary function test, the hospital anxiety and depression scale (HADS), quality of life (QOL) assessment, and many others.

Future prospective randomized controlled clinical trials are needed to confirm that hand-assisted sputum excretion can effectively reduce PPCs of esophageal cancer.

\section{Acknowledgments}

Thanks to AME for helping with the article language. Funding: None.

\section{Footnote}

Reporting Checklist: The authors have completed the STROBE reporting checklist. Available at http://dx.doi. org/10.21037/apm-20-1267

Data Sharing Statement: Available at http://dx.doi. org/10.21037/apm-20-1267

Conflicts of Interest: All authors have completed the ICMJE uniform disclosure form (available at http://dx.doi. org/10.21037/apm-20-1267). The authors have no conflicts of interest to declare.

Ethical Statement: The authors are accountable for all aspects of the work in ensuring that questions related to the accuracy or integrity of any part of the work are appropriately investigated and resolved. The study was conducted in accordance with the Declaration of Helsinki (as revised in 2013). The study protocol was approved by the Ethics Committee of the Affiliated Cancer Hospital of Zhengzhou University (2014ys38), and the requirement for informed consent was waived because of the retrospective nature of the research.

Open Access Statement: This is an Open Access article distributed in accordance with the Creative Commons Attribution-NonCommercial-NoDerivs 4.0 International License (CC BY-NC-ND 4.0), which permits the noncommercial replication and distribution of the article with the strict proviso that no changes or edits are made and the original work is properly cited (including links to both the formal publication through the relevant DOI and the license).
See: https://creativecommons.org/licenses/by-nc-nd/4.0/.

\section{References}

1. Guinan EM, Bennett AE, Doyle SL, et al. Measuring the impact of oesophagectomy on physical functioning and physical activity participation: a prospective study. BMC Cancer 2019;19:682.

2. Zhang H, Yang $Y$, Shang $Q$, et al. Predictive value of preoperative weight loss on survival of elderly patients undergoing surgery for esophageal squamous cell carcinoma. Transl Cancer Res 2019;8:2752-8.

3. Seesing MFJ, Kingma BF, Weijs TJ, et al. Reducing pulmonary complications after esophagectomy for cancer. J Thorac Dis 2019;11:S794-8.

4. Derogar M, Orsini N, Sadr-Azodi O, et al. Influence of major postoperative complications on health-related quality of life among long-term survivors of esophageal cancer surgery. J Clin Oncol 2012;30:1615-9.

5. Okamura A, Watanabe M, Mine S, et al. Spirometric Lung Age Predicts Postoperative Pneumonia After Esophagectomy. World J Surg 2016;40:2412-8.

6. Klevebro F, Elliott JA, Slaman A, et al. Cardiorespiratory Comorbidity and Postoperative Complications following Esophagectomy: a European Multicenter Cohort Study. Ann Surg Oncol 2019;26:2864-73.

7. Nozaki I, Mizusawa J, Kato K, et al. Impact of laparoscopy on the prevention of pulmonary complications after thoracoscopic esophagectomy using data from JCOG0502: a prospective multicenter study. Surg Endosc 2018;32:651-9.

8. Guinan EM, Dowds J, Donohoe C, et al. The physiotherapist and the esophageal cancer patient: from prehabilitation to rehabilitation. Dis Esophagus 2017;30:1-12.

9. van der Sluis PC, Grimminger PP, van Hillegersberg $\mathrm{R}$, et al. Hybrid minimally invasive esophagectomy for esophageal cancer: less is more. J Thorac Dis 2019;11:S1935-7.

10. Nakatsuchi T, Otani M, Osugi H, et al. The necessity of chest physical therapy for thoracoscopic oesophagectomy. J Int Med Res 2005;33:434-41.

11. Guinan EM, Forde C, O'Neill L, et al. Effect of preoperative inspiratory muscle training on physical functioning following esophagectomy. Dis Esophagus 2019;32:doy091.

12. Katsura M, Kuriyama A, Takeshima T, et al. Preoperative inspiratory muscle training for postoperative pulmonary 
complications in adults undergoing cardiac and major abdominal surgery. Cochrane Database Syst Rev 2015;(10):CD010356.

13. Ward TJC, Plumptre CD, Dolmage TE, et al. Change in $\mathrm{V}$ O2peak in Response to Aerobic Exercise Training and the Relationship With Exercise Prescription in People With COPD: A Systematic Review and Meta-analysis. Chest 2020;158:131-44.

14. Hanada M, Kanetaka K, Hidaka S, et al. Effect of early mobilization on postoperative pulmonary complications in patients undergoing video-assisted thoracoscopic surgery on the esophagus. Esophagus 2018;15:69-74.

15. Fagevik Olsén M, Hahn I, Nordgren S, et al. Randomized controlled trial of prophylactic chest physiotherapy in major abdominal surgery. Br J Surg 1997;84:1535-8.

16. Futier E, Paugam-Burtz C, Godet T, et al. Effect of early postextubation high-flow nasal cannula vs conventional oxygen therapy on hypoxaemia in patients after major abdominal surgery: a French multicentre randomised controlled trial (OPERA). Intensive Care Med 2016;42:1888-98.

17. Feng F, Ji G, Li JP, et al. Fast-track surgery could improve postoperative recovery in radical total gastrectomy patients. World J Gastroenterol 2013;19:3642-8.

18. Barbagallo M, Ortu A, Spadini E, et al. Prophylactic use of helmet CPAP after pulmonary lobectomy: a prospective randomized controlled study. Respir Care 2012;57:1418-24.

19. Kindgen-Milles D, Muller E, Buhl R, et al. Nasalcontinuous positive airway pressure reduces pulmonary morbidity and length of hospital stay following thoracoabdominal aortic surgery. Chest 2005;128:821-8.

20. Yang M, Ahn HJ, Kim K, et al. Does a protective ventilation strategy reduce the risk of pulmonary complications after lung cancer surgery?: a randomized controlled trial. Chest 2011;139:530-7.

21. Treschan TA, Schaefer M, Kemper J, et al. Ventilation with high versus low peep levels during general anaesthesia for open abdominal surgery does not affect postoperative spirometry: A randomised clinical trial. Eur J Anaesthesiol 2017;34:534-43.

22. Rigg JR, Jamrozik K, Myles PS, et al. Epidural anaesthesia and analgesia and outcome of major surgery: a randomised trial. Lancet 2002;359:1276-82.

23. Zhu Z, Wang C, Xu C, et al. Influence of patientcontrolled epidural analgesia versus patient-controlled intravenous analgesia on postoperative pain control and recovery after gastrectomy for gastric cancer: a prospective randomized trial. Gastric Cancer 2013;16:193-200.

24. Odor PM, Bampoe S, Gilhooly D, et al. Perioperative interventions for prevention of postoperative pulmonary complications: systematic review and meta-analysis. BMJ 2020;368:m540.

25. Gan TJ, Soppitt A, Maroof M, et al. Goal-directed intraoperative fluid administration reduces length of hospital stay after major surgery. Anesthesiology 2002;97:820-6.

26. Benes J, Chytra I, Altmann P, et al. Intraoperative fluid optimization using stroke volume variation in high risk surgical patients: results of prospective randomized study. Crit Care 2010;14:R118.

27. Nakamura M, Iwahashi M, Nakamori M, et al. An analysis of the factors contributing to a reduction in the incidence of pulmonary complications following an esophagectomy for esophageal cancer. Langenbecks Arch Surg 2008;393:127-33.

28. Ford SJ, Adams D, Dudnikov S, et al. The implementation and effectiveness of an enhanced recovery programme after oesophago-gastrectomy: a prospective cohort study. Int J Surg 2014;12:320-4.

29. Low DE, Allum W, De Manzoni G, et al. Guidelines for Perioperative Care in Esophagectomy: Enhanced Recovery After Surgery (ERAS®) Society Recommendations. World J Surg 2019;43:299-330.

30. Valkenet K, Trappenburg JCA, Ruurda JP, et al. Multicentre randomized clinical trial of inspiratory muscle training versus usual care before surgery for oesophageal cancer. Br J Surg 2018;105:502-11.

31. Pfirrmann D, Tug S, Brosteanu O, et al. Internet-based perioperative exercise program in patients with Barrett's carcinoma scheduled for esophagectomy [iPEP - study] a prospective randomized-controlled trial. BMC Cancer 2017;17:413.

32. Castelino T, Fiore JF Jr, Niculiseanu P, et al. The effect of early mobilization protocols on postoperative outcomes following abdominal and thoracic surgery: A systematic review. Surgery 2016;159:991-1003.

33. Lunardi AC, Cecconello I, Carvalho CR. Postoperative chest physical therapy prevents respiratory complications in patients undergoing esophagectomy. Rev Bras Fisioter 2011;15:160-5.

34. Selsby D, Jones JG. Some physiological and clinical aspects of chest physiotherapy. Br J Anaesth 1990;64:621-31.

35. Lewis LK, Williams MT, Olds TS. The active cycle of breathing technique: a systematic review and metaanalysis. Respir Med 2012;106:155-72. 
36. Law S, Wong KH, Kwok KF, et al. Predictive factors for postoperative pulmonary complications and mortality after esophagectomy for cancer. Ann Surg 2004;240:791-800.

37. Ferguson MK, Celauro AD, Prachand V. Prediction of major pulmonary complications after esophagectomy. Ann Thorac Surg 2011;91:1494-500; discussion 1500-1.

38. Goense L, Meziani J, Bulbul M, et al. Pulmonary diffusion capacity predicts major complications after esophagectomy for patients with esophageal cancer. Dis Esophagus 2019;32:doy082.

39. Wynter-Blyth V, Moorthy K. Prehabilitation: preparing patients for surgery. BMJ 2017;358:j3702.

40. Doganay E, Moorthy K. Prehabilitation for esophagectomy. J Thorac Dis 2019;11:S632-8.

41. Findlay JM, Gillies RS, Millo J, et al. Enhanced recovery for esophagectomy: a systematic review and evidencebased guidelines. Ann Surg 2014;259:413-31.

42. Bhatt NR, Sheridan G, Connolly M, et al. Postoperative exercise training is associated with reduced respiratory infection rates and early discharge: A case-control study.

Cite this article as: Wang W, Liu Q , Yu Y, Ma H, Xu L, Zhang R, Sun H, Wang Z, Zheng Y, Chen P, Liu S, Yang F, Zou Q, Sun A, Chu X, Gong C, Xing W. Hand-assisted sputum excretion can effectively reduce postoperative pulmonary complications of esophageal cancer. Ann Palliat Med 2020;9(6):3721-3730. doi: 10.21037/apm-20-1267
Surgeon 2017;15:139-46.

43. Fujimoto S, Nakayama T. Effect of combination of preand postoperative pulmonary rehabilitation on onset of postoperative pneumonia: a retrospective cohort study based on data from the diagnosis procedure combination database in Japan. Int J Clin Oncol 2019;24:211-21.

44. Bolger JC, Loughney L, Tully R, et al. Perioperative prehabilitation and rehabilitation in esophagogastric malignancies: a systematic review. Dis Esophagus 2019;32:doz058.

45. Granger CL. Physiotherapy management of lung cancer. J Physiother 2016;62:60-7.

46. Bender T. Evidence-based physiotherapy. Orv Hetil 2013;154:1893-9.

47. Remondi Re R, Durafourg MP. A new look at physiotherapy in France in 2018. Sante Publique 2018;30:869-76.

(English Language Editor: J. Gray) 